\section{La "arquitectura" de la COVID-19}

\section{Francesc Pernas Gali}

Recibido 2020.04.07 :.: Aceptado 2020.04.13 DOI: 10.5821/palimpsesto.21.949

Persona de contacto: fpernas@casasolo.es ORCID: https://orcid.org/0000-0003-0503-4755 Arquitecto por la ETSAB

\section{ABSTRACT}

En el momento en que se escriben estas reflexiones, a primeros del mes de abril de 2020, la pandemia que afecta a buena parte del planeta es molvo de rellexión en todos los ámbitos.

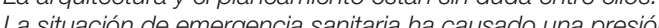
muy fuerte en los sistemas y las infraestructuras sanitarias. Los hospitales han respondido bion, gracias al el esfuerzo imaginación de los profesionales que los utilizan. El personal es el único recurso que no se puede fabricar. ni construirPara apoyar y proporcionar más espacio a los hospitales ya establecidos se ha recurrido a la reutilización de edificios ya establecidos se ha recurrido a la reutilización de edfif han empleado el término "arquitectura" para definir estas actuaciones. En el curso de una tragedia es necesario habilitar soluciones de emergencia, imprescindibles para sobrevivir, pero no disponen de ninguna de las cualidades de la arquitectura hospitalaria actual, ni para los pacientes, ni para quienes trabajan allí. Serían, en palabras de Nikolaus Pevsner, "cobertizos para bicicletas".

La emergencia debe ser la excepción. Hay que trabajar para mejorar la respuesta cuando se produce, y la experiencia obtenida en cada uno de sus episodios se debe utilizar para avanzar, aún con más rapidez, en las lineas de investigación ya abiertas en la arquitectura sanitaria. La "arquitectura" de la COVID-19 no ha venido para quedarse. No se puede hacer una arquitectura para cada enfermedad. Eso corresponde a siglos pasados. La arquitectura, los hospitales también le pertenecen, forma parte de la cultura de un país, debe preservarse, y en momentos como este no debe olvidarse.

PALABRAS CLAVE: arquitectura sanitaria; covid-19; emergencias; planificación; innovación$$
\text { orprendió a todo el mundo, arquitecto }
$$
incluidos, que en Wuhan 7.000 trabajadores empezaran a construir el Hospital Huoshenshan

de 1000 camas, que iba a estar terminado en 10 días. En otro distrito de Wuhan, de forma solapada, iniciaron la construcción del Hospital Lieshenshan de 1.600 camas, que entró en funcionamiento una semana más tarde. China ya tenía experiencia en este tipo de situaciones. En 2003, para combatir la epidemia de SARS-CoV, en Beijing se había construido un hospital en sólo 7 días. Al principio, cuando el Dr. Li Wanliang detectó varios casos de una neumonía atípica, las autoridades no le prestaron la atención debida. Pero desde el 20 de enero, en que se declaró el estado de emergencia y se confinó a toda la población de la provincia de Hubei, China tomó decisiones acertadas para controlar la epidemia.

La epidemia se transformó en pandemia. Apenas dos meses más tarde, un nuevo coronavirus muy agresivo de alta morbilidad, ya identificado como SARS-CoV-2, se ha diseminado por dos centenares de países de todo el mundo. Tarde y mal, en muchos de ellos, se han establecido restricciones de movilidad y controles insuficientes en muchos casos, que han conducido a un estrés de sus sistemas sanitarios jamás experimentado hasta ahora. Las medidas tomadas, y sus consecuencias de todo tipo, no se podrán evalua hasta que se consiga el objetivo primordial de control de la pandemia. Las formas de combatirla han sido diversas, según las estructuras políticas, sociales, económicas y sanitarias de cada estado.

Sería una temeridad no aprender de las lecciones recibidas, y no establecer los medios y las soluciones necesarias para evitar en el futuro los errores cometidos. La toma de decisiones deberá hacerse mediante un consenso amplio, tanto a nivel local como global.

La pandemia, que afecta de modo más severo a las zonas urbanas, probablemente conducirá a un debate sobre su densidad y planificación. El confinamiento también debe proporcionar datos sobre el uso y carencias de los diversos tipos actuales de vivienda, tanto individual como colectiva. Se requerirán aportaciones desde todas las disciplinas. La urbanística y la arquitectura están, sin duda, entre ellas.

A estas dos disciplinas, y especialmente desde la planificación sanitaria y el diseño de hospitales, les he dedicado la mayor parte de mi trabajo hasta mi retirada de la primera línea, hace unos años. Desde el confinamiento obligado, y admitido por convicción, he sentido la necesidad de compartir algunas reflexiones, centradas en esa parcela específica del conocimiento.

En estos momentos de autodefensa contra la pandemia, la mayoría de los países afectados - y también aquellos que aún no han experimentado la COVID-19 con toda su crudeza - están abocando los máximos recursos para reforzar sus infraestructuras de salud, tal como hicieron en China hace ya más de dos meses. Se están instalando o realizando construcciones de todo tipo para apoyar a los hospitales y centros sanitarios ya establecidos.

En diarios y publicaciones especializadas, aparecen reportajes y artículos sobre cómo se organizan estos dispositivos en diversos países. Al leerlos, me llamó la atención que a menudo se emplee el término arquitectura para referirse a ellos. ${ }^{1}$ Lo primero que me vino a la mente fue la famosa frase escrita por $\mathrm{e}$ historiador y crítico de arte Sir Nikolaus Pevsner: "A bicycle shed is a building; Lincoln Cathedral is a piece of architecture". ${ }^{2}$ La frase de Pevsner me permite distinguir entre dos vías de trabajo relativas a los hospitales que, siendo a lo mejor complementarias, debemos mantener vivas en el futuro. E incluso mucho más activas.

Es evidente que los cobertizos para bicicletas son hoy tan necesarios, y alguien diría que incluso más, que las catedrales. Y según qué cobertizo para bicicletas también podría ser considerado como arquitectura Pero lo que en este momento creo que es interesante subrayar es la existencia de dos líneas de investigación que no se deben confundir. En una tragedia como la que se está viviendo en todo el mundo es imprescindible habilitar soluciones de emergencia. Son, en general, "cobertizos de bicicletas", necesarios para poder sobrevivir en estos imprevisibles momentos de crisis.

Esa es la primera línea de trabajo que debe plantearse una vez se recupere la actividad de las empresas, que actualmente se ve mermada por disposiciones y decisiones colectivas, tomadas en beneficio de la salud pública. En el caso de China, la réplica del Hospital Xiaotangshan que se construyó en Wuhan en sólo diez días, fue un "cobertizo" que ya venía muy trabajado de experiencias anteriores, como el SARS de 2003. Ahí es donde entra la planificación. Se deberán planificar en las ciudades espacios libres para situaciones de emergencia, cercanos a los hospitales. Los solares a ellos destinados también deberán disponer de espacios de reserva. En zonas urbanas densamente pobladas, la existencia en los alrededores de pabellones o instalaciones deportivas, podría cumplir la misma función que las zonas libres. A parte de las instalaciones y dispositivos militares, casi siempre precarios aunque de gran ayuda en muchos casos, se deben destinar recursos e imaginación a potenciar soluciones civiles más humanizadas. Aún en circunstancias tan dramáticas como las actuales, y las que especialmente suceden en el norte de Italia, son de destacar algunas iniciativas como la del hospital de la Feria de Milano ${ }^{3}$.

También en Milan, la iniciativa de diseño para la reutilización de contenedores como módulos de aislamiento realizada por CRA Associati ${ }^{4}$ (FIG. 1), es de carácter menos local y más global. Iniciativas de este tipo, o como los módulos hinchables que fabrica Tecnodimensión en Girona, podrían ser impulsadas por organismos internacionales, incluso para mejorar la gestión presente de algunos programas de Naciones Unidas. Para ello será necesario contar con profesionales sanitarios, ingenieros y arquitectos, especialmente preparados.

La segunda línea de trabajo debería evitar ser contaminada por la anterior como producto de la urgencia y de las prisas, pues correría el peligro de debilitarse. Es la de los nuevos hospitales, o los ya existentes que puedan tener previstas renovaciones o ampliaciones futuras. Es lo que, salvando la discusión de las bicicletas y la iglesia, se entiende por arquitectura hospitalaria. Incluso en la China Popular, se sabe distinguir entre un hospital de campaña y un hospital como el Gulou de Nanjing ${ }^{5}$, por ejemplo (FIG. 2).

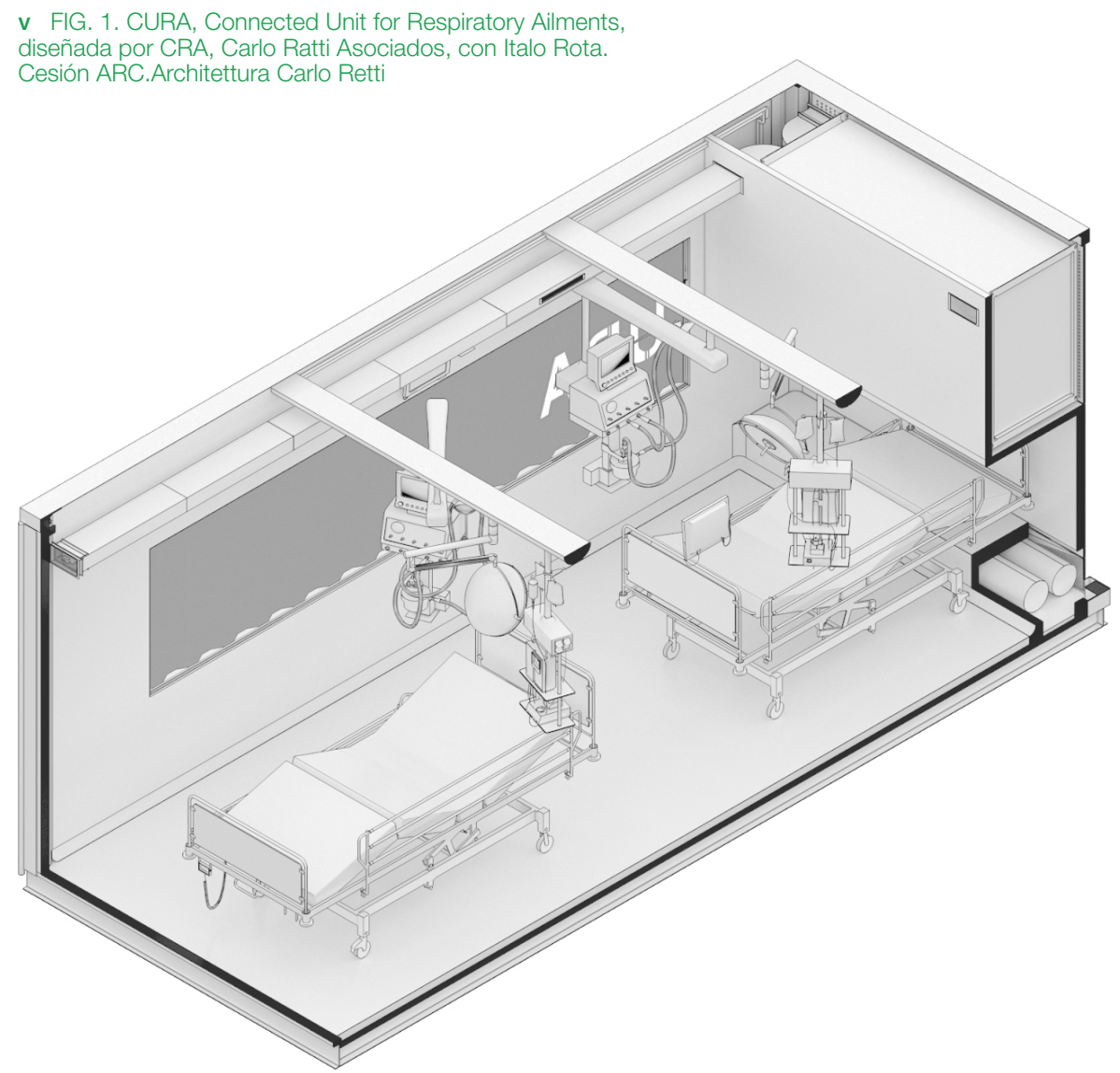




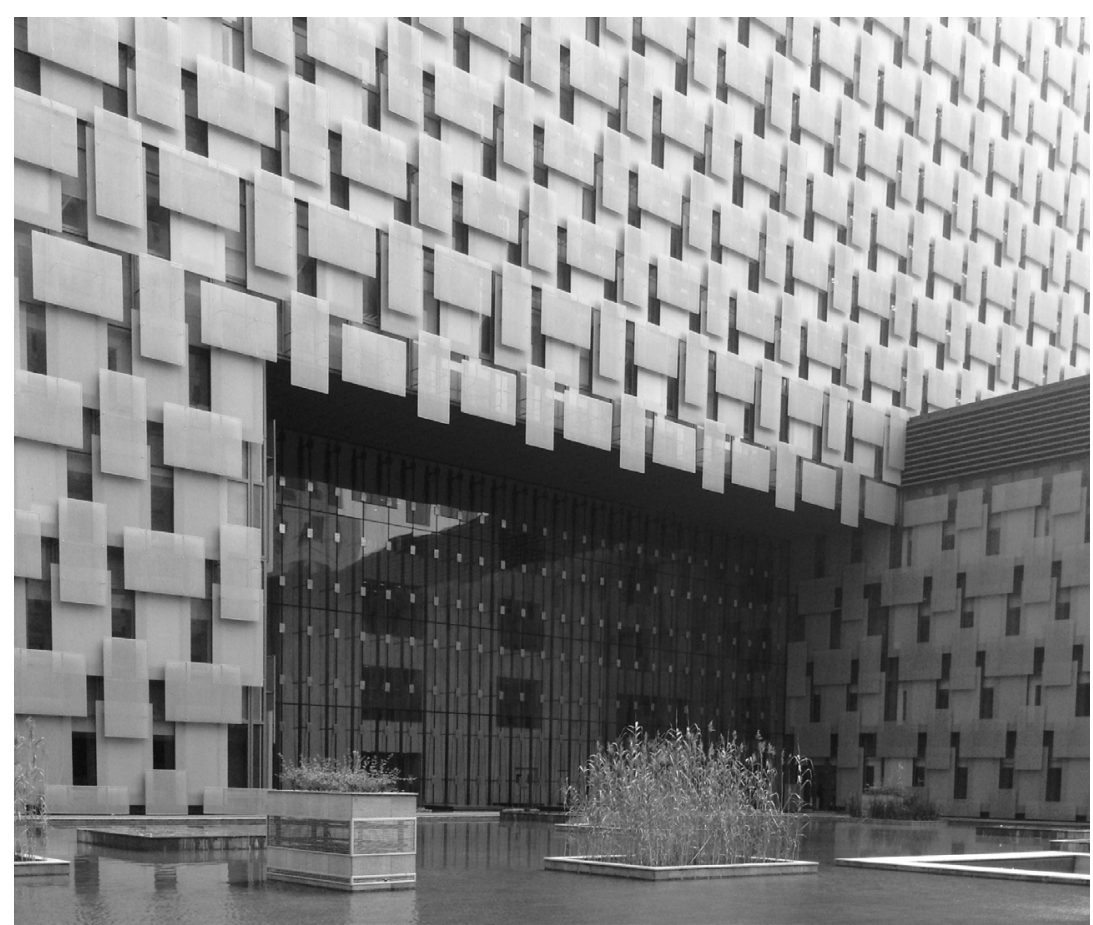
FIG. 2. Drum Tower del Hospital Gulou de Nanjing, Lemanarc SA, arquitectos. 2012
Fotografía del autor

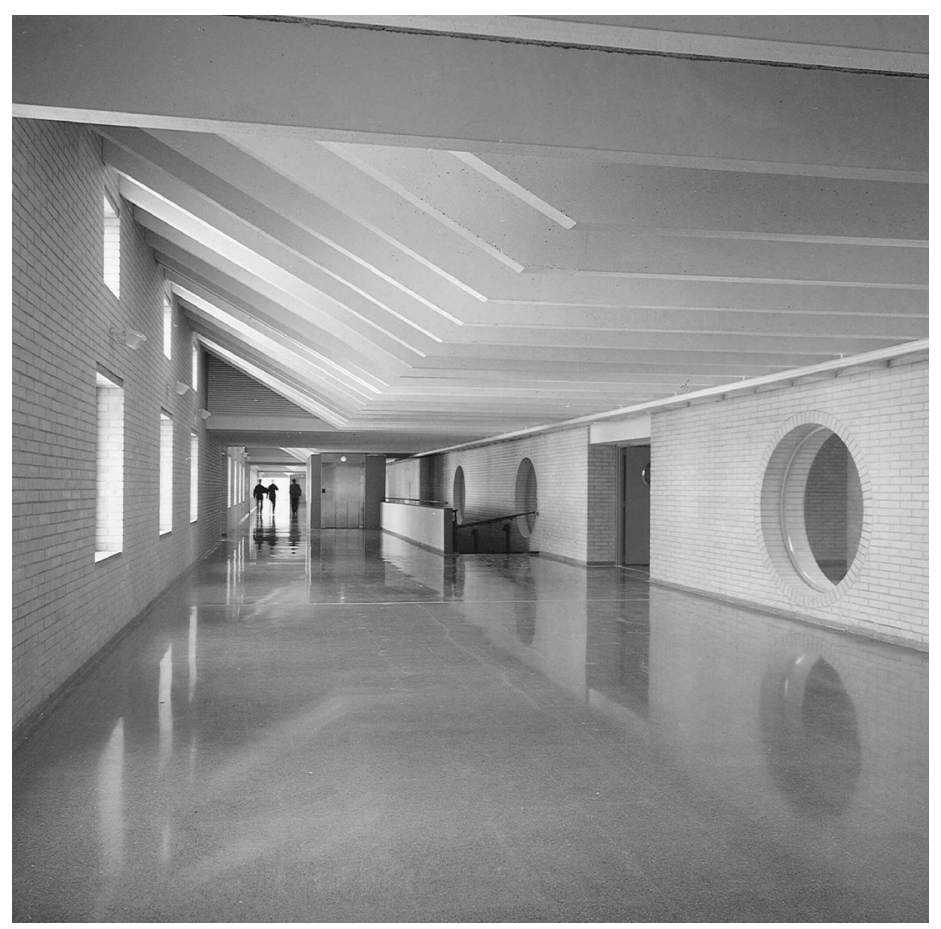

FIG. 3. Hospital street, o espina, del Hospital Comarcal del Alt Penedés, Casa Sólo Arquitectos. Cesión Casa Solo Arquitectos SLP
Después de la Guerra Civil, en una situación de reconstrucción y de recuperación económica del país, el INSALUD de forma centralizada, y sobre todo a parti de los años Sesenta, construyó en España algunos "cobertizos para bicicletas". Casi siempre se levantaron en poco más de seis meses. Cumplieron con su cometido; pero eran hospitales de campaña - de hecho lo eran, pero de otro tipo - y no creo que pudieran considerarse arquitectura. Además, esos "hospitales kleenex" de franjas azules y blancas, dan muchos problemas de mantenimiento y reparación como ha podido comprobar la Generalitat de Catalunya, desde que asumió las competencias en 1981.

Con el progresivo traspaso de competencias, en todas las Comunidades Autónomas casi siempre se recuperó la arquitectura. Disponemos en nuestro país de excelentes arquitectos, y no sólo especialistas, que han hecho de los hospitales españoles un referente a nivel europeo. No hace falta justificar aquí el papel positivo de la arquitectura en las actividades humanas. Cualquier sociedad avanzada sabe distinguir, o debe, lo que las raíces históricas, culturales y sociales le aportan.

De la situación actual de carga intensiva a que los hospitales están sometidos debido a la pandemia será necesario extraer experiencia para aplicarla en el diseño futuro. Algunas ideas de partida, tales como:

a) Ambientes cada vez más humanizados, confort, luz natural, domesticidad, dado que las estancias medias posiblemente tenderan a incrementarse en el futuro.

b) Zonas exteriores o terrazas donde los pacientes, y también el personal, puedan recuperar fuerzas.

c) Los espacios amplios y diáfanos, como grandes salas de espera, vestíbulos o "hospital streets" deberán dotarse de tomas clínicas para poder acoger camas ${ }^{6}$ (FIG. 3).

d) Las dimensiones de las habitaciones estándar permitirán intubar a un paciente.

e) Sistemas adicionales de comunicaciones centralizados que permitan convertir camas estándar en puntos de cuidados intensivos controlados a distancia, sólo con aportación de los equipos necesarios (monitores, bombas de perfusión, respiradores, ...).

Conjuntos fáciles de ser aislados, tanto en las instalaciones como en lo físico.

g) Edificios cercanos, o integrados en el conjunto, que puedan ser utilizados como residencia.

h) Evitar que se ocupen espacios diáfanos, tanto interiores como exteriores, mediante actuaciones no programadas. Necesidad de planificación actualizada, mediante planes directores, que prevean situaciones catastróficas o de epidemias.

i) Priorizar, siempre que se pueda, soluciones bajas y "open-ended" frente a las concentradas.

Circulaciones horizontales, incluso sin diferenciar, frente a la multiplicidad de ascensores.

k) Estudio e implantación de sistemas robóticos.

1) Priorizar la conectividad exterior 5G, a nivel de profesionales y también de los pacientes, sobre todo con el exterior en casos de aislamiento estricto.

m) Aprovechar el diseño Age-friendly Hospitals para modificar la percepción que los mayores van a tener de los hospitales como lugares inhóspitos y peligrosos.

as residencias de la tercera edad no deben quedar al margen de los sistemas de salud. La cruda realidad actual así lo aconseja. No se pueden limitar sólo a lo social. Muchas de las ideas que surjan para los centros sanitarios también se les deben aplicar. En ellas, en momentos de infecciones que provienen del exterior se ha comprobado que el mejor remedio es confinamiento, incluso de los integrantes del personal que lo aceptasen. Esas contingencias, a partir de ahora, deberán estar previstas.

Los hospitales están respondiendo muy bien aumentando su capacidad mediante reorganizaciones internas, con la imaginación y el esfuerzo personal de los equipos de profesionales que los están utilizando. Especializar hospitales, una solución que emula los antiguos hospitales de Infecciosos, o habilitar dispositivos de soporte como los hoteles que tienen gran cantidad de habitaciones, complementan el esfuerzo de quienes están en contacto directo con el paciente. Pero sin personas y equipos, no sirven para nada.

Primero las personas, por ese orden. Es evidente que con las infraestructuras no basta. El personal que atendió a los pacientes del hospital construido en 10 días junto a Vulcan Hill en Wuhan, era persona sanitario procedente del Ejército Popular, unos 1400 médicos. Sin personal, el hospital era imposible que funcionase. El personal es el único recurso que no se puede fabricar. Es insustituible, no son máquinas. Debe cuidarse mucho más que ellas. La formación y preparación para casos como el presente, que pueden ser cada vez más frecuentes, es indispensable. La consideración que merece todo el personal, sanitario y no sanitario, que atiende a los dispositivos de salud, ya no sólo económica, también es primordial.

En segundo lugar, los equipos. El sistema debe estar preparado y tener reservas, o la capacidad estratégica de conseguirlas en un corto espacio de tiempo, para enfrentarse a situaciones como la actual u otras parecidas. Veremos si a partir de este momento las administraciones y los gobiernos priorizan aquellos sectores y empresas que se han mostrado imprescindibles, o aquellas otras que hemos echado en falta por permitir que desaparecieran. O que desaparecerán si después de esto se sigue priorizando lo de siempre y no se llevan a cabo planes de rescate. El BCE, si es que Europa existe, debe poner en marcha las rotativas, aunque ello pudiera suponer una devaluación del euro.

La arquitectura forma parte de la cultura de un país, y debe preservarse. La defensa de la cultura en situaciones como la presente, no debe olvidarse. Nos ayuda a permanecer en nuestras casas beneficiándonos del poder estabilizador de la música, el arte, la literatura, o la poesía. Un país con personas más educadas y responsables, necesitaría menos controles para mantener la salud pública. Ya lo decía esa pintada anarco que corría allá por los Sesenta del siglo pasado: "Más poesía y menos policía". Pero ello requiere estructuras muy democráticas, y establecer políticas más inteligentes y a largo plazo.. Este no es el lugar para debatirlo, aunque es apremiante.

1 Véase por ejemplo: "Cinco arquitecturas urgentes y efímeras para combatir el Coronavirus", Diario El Mundo, 1 de abril de 2 "Un cobertizo para bicicletas es una construcción; la Nikolaus Pevsner (1902-1983), An outline of European architecture, 1945 p.XVI.

${ }^{3}$ El 31 de marzo se puso en marcha la primera fase del Ospedale Fiera Milano, como anexo dependiente del Policlínico en 10 días. "... No hicimos un hospital de campaña como en el pasado, sino un hospital especializado, con respeto para el paciente y los trabajadores de la salud..." (Mensaje de Guido Bertolaso, asesor de la Lombardía para la emergencia del coronavirus).

${ }^{4}$ CURA, Connected Unit for Respiratory Ailments, diseñada por CRA, Carlo Ratti Asociados, con Italo Rota Contenedores de 6×2,4×2,6m.reutilizados como módulos de aislamiento conectables, de los que se está produciendo un prototipo financiado por UniCredit. Ver FIG.

5 Drum Tower del Hospital Gulou de Naniing, Lemanarc SA arquitectos. 2012. Ver FIG. 2.

${ }^{6}$ Hospital street, o espina, del Hospital Comarcal del Alt Penedés, Casa Sólo Arquitectos. Un ejemplo con capacidad para que se aplique esta estrategia. Ver FG. 3.

FRANCESC PERNAS GAĹ es arquitecto y presidente de Casa Solo Arquitectos. 